\title{
Symposium 5
}

\section{Interaction between Blood Cells and Vascular Wall}

\section{Endothelial-Dependent Mechanisms of Leukocyte Adhesion*}

\author{
Michael A. Gimbrone Jr.**, M. Elyse Wheeler** \\ and Michael P. BeVILACQUA**
}

\begin{abstract}
Introduction
Localized adhesion of circulating leukocytes to the blood vessel wall is an essential component of inflammatory reactions and various vascular disease processes, including vasculitis, allograft rejection and atherosclerosis. There is increasing evidence that vascular endothelial cells play an active role in this process. Our research group has been especially interested in defining endothelialdependent mechanisms of leukocyte adhesion, and exploring their relevance for human vascular pathophysiology. The following is a brief account of our recent progress in characterizing inducible endothelial cell surface structures involved in leukocyte adhesion ("endothelial-leukocyte adhesion molecules, ELAMs") and a soluble endothelial-derived leukocyte adhesion inhibitor ("LAI").
\end{abstract}

Endothelial-leukocyte adhesion molecules: ELAMs

As an experimental model, our laboratory has used cultured human endothelial cells $(\text { HEC })^{1}$, in standardized monolayer adhesion assays ${ }^{2)}$, to study factors that can alter leukocyte-endothelial adhesion and to explore the molecular mechanisms involved. In particular, we have examined the hypothesis that certain inflammatory substances can act directly on vascular endothelial cells to increase the adhesion of blood leukocytes ${ }^{3)}$. We initially found that human monocyte-derived IL-1 (IL-1) could act on cultured HEC in a time- and protein synthesis-dependent fashion to increase the adhe-

* This paper was presented at the winter meeting of the Japan Atherosclerosis Society in Japan in December 1987

** Vascular Research Division, Department of Pathology, Brigham and Women's Hospital and Harvard Medical School, Boston, MA, U.S.A. sion of isolated human polymorphonuclear leukocytes (PMN), monocytes, and the related cell lines HL-60 and U937 ${ }^{3,4}$. Subsequently, we studied the effects of other cytokines, including recombinant IL-1-alpha, IL-1-beta, tumor necrosis factor (TNF), and lymphotoxin (LT), as well as bacterial endotoxin (lipopolysaccharide (LPS) $)^{5,6}$. Other laboratories have made similar observations using various mediators and leukocyte cell types ${ }^{7-12}$. Together, these studies suggested that "activation" of vascular endothelial cells by certain stimuli results in increased expression of cell surface adhesive molecules that can bind blood leukocytes. We have referred to these putative structures as "endothelial-leukocyte adhesion molecules" (ELAMs).

The following experimental observations are consistent with the existence of inducible ELAMs: (1) Stimulation of endothelial adhesiveness for leukocytes by cytokines or endotoxin is a timedependent process that requires protein and RNA synthesis as defined by blocking with cycloheximide and actinomycin D. However, inhibition of arachidonate metabolism, via the cyclooxygenase pathway, has no effect on this phenomenon; (2) The generation of soluble mediators by IL-1-treated HEC does not appear to be essential for the augmentation of leukocyte adhesion, since IL-1stimulated HEC conditioned medium was routinely removed before the adhesion assay. In fact, we have recently found that conditioned media from IL-1-treated HEC contain an inhibitor of endothelial-PMN adhesion (see below); (3) Increased adhesiveness is maintained following brief paraformaldehyde fixation of IL-1-treated endothelial monolayers, suggesting that ongoing cellular metabolism is not required; (4) In subconfluent monolayers, increased adhesion is observed microscopically over the surface of individual cells, not 
in the intervening noncellular spaces. To test the "ELAM Hypothesis" directly and to better define cytokine-induced alterations in endothelial cell surface structures, we have developed monoclonal antibodies to IL-1- and TNF-treated endothelial cells.

\section{Identification of endothelial-leukocyte adhesion molecule-1 (ELAM-1)}

We have characterized two monoclonal antibodies, designated $\mathrm{H} 4 / 18$ and $\mathrm{H} 18 / 7$, which recognize an endothelial cell surface protein expressed on cytokine or endotoxin treated HEC, but not on unstimulated $\mathrm{HEC}^{5,6,13)}$. The same mediators that act on HEC to increase leukocyte adhesion (e.g., IL-1, TNF, LT, LPS) also induce the expression of the epitopes recognized by Mab H4/18 and Mab H18/7. Peak HEC expression of these epitopes occurs after 4 hours stimulation and correlates with peak expression of HEC adhesiveness for leukocytes. Other cytokines, including interferon-alpha, -beta or -gamma, and IL-2, did not stimulate leukocyte adhesion or monoclonal antibody binding. All other cell types tested were found to be negative for Mab H18/7 and Mab $\mathrm{H} 4 / 18$ binding by flow cytometry or surface radioimmunoassay, including PMN, blood mononuclear leukocytes and HL-60 cells, before and after stimulation with phorbol ester, as well as cultured human dermal fibroblasts, before and after incubation with IL-1.

Immunoprecipitation from total cell extracts of metabolically labeled IL-1 or TNF-stimulated HEC with Mab H18/7 and Mab H4/18 yielded two polypeptides (major species, $\mathrm{Mr}$ 115,000; minor species, $\mathrm{Mr}$ 95,000) which were not detected in unstimulated $\mathrm{HEC}^{6}$ ). Exhaustive treatment of HEC lystates by Mab H18/7 removed all Mab $\mathrm{H} 4 / 18$ reactive polypeptides and vice-versa, thus indicating that these two monoclonal antibodies were recognizing the same molecule in cytokine activated HEC.

When tested in leukocyte adhesion assays, Mab H4/18 partially (15-30\%) blocked HL-60 cell adhesion to IL-1-activated $\mathrm{HEC}^{5}$, but failed to inhibit PMN adhesion ${ }^{6}$. In contrast, Mab H18/7 significantly and consistently blocked the adhesion of both HL-60 cell (more than $60 \%$ ) and PMN (more than $50 \%$ ) to IL-1-activated $\mathrm{HEC}^{6}$ ). In contrast, neither monoclonal antibody appears to be effective in blocking peripheral blood monocyte or lymphocyte adhesion to HEC. Based on the ability of Mab H18/7 to block the adhesion of PMN and HL-60 cells to activated HEC, we have designated the structure recognized by this antibody as "endothelial-leukocyte adhesion molecule 1 (ELAM-1)"6).

Several characteristics distinguish ELAM-1 from previously identified cell surface adhesive structures, such as platelet glycoprotein IIB/IIIalike proteins, intercellular adhesion molecule 1 (ICAM-1) ${ }^{14)}$, and HLA-DR ${ }^{15)}$, which may be expressed on endothelial cells. These characteristics include: (1) The lack of basal (unstimulated) endothelial expression of ELAM-1; (2) the mediator specificity and kinetics of its induction; and (3) its molecular weight characteristics. Definitive characterization of ELAM-1 and clarification of its potential relatedness to other adhesion proteins await determination of primary structure, now in progress.

In summary, we have demonstrated that activation of cultured human vascular endothelium by inflammatory-immune mediators, such as IL-1, TNF, LT, and LPS, results in the expression of a novel surface molecule, ELAM-1, which can bind PMN, HL-60 cells, and possibly other leukocyte types. Using these monoclonal antibodies to immunohistochemically stain human tissues, microvascular endothelium has been shown to express ELAM-1 transiently during certain pathologic processes ${ }^{16)}$. We propose that modulation of ELAM-1 expression may contribute to the regulation of leukocyte-vessel wall interactions in a variety of pathophysiologic settings.

\section{An endothelial-derived leukocyte adhesion inhibitor (LAI)}

In addition to this pro-adhesive cell surface change, cytokine and LPS stimulated HEC cultures also appear to generate a soluble anti-adhesive activity, a "leukocyte adhesion inhibitor" (LAI) $)^{17-19)}$. Conditioned media collected from IL-1-treated HEC cultures (but not from sham-treated cultures), act selectively on PMN to inhibit their adhesion to activated HEC monolayers. Production of LAI is blocked by prior treatment with protein or RNA synthesis inhibitors, but not by treatment with aspirin or other cyclooxygenase inhibitors. Preliminary characterization indicates that LAI is nonsedimentable $(250,000 \times \mathrm{g}, 45 \mathrm{~min})$, nondialyzable, and stable to heat $\left(80^{\circ} \mathrm{C}, 30 \mathrm{~min}\right)$ 
and acid treatment. LAI can be precipitated with ammonium sulfate and is destroyed by pepsin treatment. LAI does not alter PMN resting membrane potential or stimulus-induced membrane depolarization, or leukotriene $\mathbf{B}_{4}$, phorbol ester or f-Met-Leu-Phe-induced changes in cytosolic free calcium levels, thus indicating that it does not produce a non-specific "toxic" effect on leukocyte function.

In selective pretreatment experiments, LAI appears to act on the leukocyte rather than on the endothelial cell. Furthermore, although LAI is an effective inhibitor of PMN adhesion, it does not appear to inhibit lymphocyte adhesion. Videomicroscopic analysis of PMN-endothelial monolayer interactions in the presence of LAI indicate that both qualitative as well as quantitative inhibitory effects are occurring. Thus, the ability of PMN to spread on the IL-1-activated endothelial surface appears to be interfered with, as well as the quantitative aspects of adhesion (i.e., reduction in the number of PMN adhering per unit area).

Further biochemical characterization of LAI and studies of the cellular and molecular mechanisms regulating its production by vascular endothelium are in progress.

\section{References}

1) Gimbrone, M. A., Jr.: Culture of vascular endothelium. Chapter 1. In: Spaet, T., ed., Progress in Hemostasis and Thrombosis, Volume 3. New York: Grune and Stratton, 1976, pp. 1-28.

2) Gimbrone, M. A., Jr. and Buchanan, M. R.: Interactions of platelets and leukocytes with vascular endothelium: in vitro studies. In: Fishman, A.P., ed., Symposium on Endothelium. New York: Annals N.Y. Acad. Sci., 1982, pp. 171-183.

3) Bevilacqua, M. P., Pober, J. S., Wheeler, M. E., Cotran, R. S. and Gimbrone, M. A., Jr.: Interleukin-1 acts on cultured vascular endothelium to increase the adhesion of polymorphonuclear leukocytes, monocytes and related cell lines. J. Clin. Invest., 76 (5): 2003-2011 (1985).

4) Bevilacqua, M. P., Pober, J. S., Wheeler, M. E., Cotran, R. S. and Gimbrone, M. A., Jr.: Interleukin 1 (IL-1) activation of vascular endothelium: Effects on procoagulant activity and leukocyte adhesion. Amer. J. Path., 121: 393-403 (1985).

5) Bevilacqua, M. P., Wheeler, M. E., Pober, J. S., Fiers, W., Mendrick, D. L., Cotran, R. S. and Gimbrone, M. A., Jr.: Endothelial-dependent mechanisms of leukocyte adhesion: Regulation by interleukin 1 and tumor necrosis factor. In: Movat, H., ed., Leukocyte Emigration and Its Sequelae, Karger, Basel, 1987, pp. 79-93.

6) Bevilacqua, M. P., Pober, J. S., Mendrick, D. L., Cotran, R. S. and Gimbrone, M. A., Jr.: Identification of an inducible endothelial-leukocyte adhesion molecule, ELAM-1. Proc. Natl. Acad Sci. U.S.A., 1987 (in press).

7) Gamble, J. R., Harlen, J. M., Klebanoff, S. J., Lopex, A. F. and Vadas, M. A.: Stimulation of the adherence of neutrophils to umbilical vein endothelium by human recombinant tumor necrosis factor. Proc. Natl. Acad. Sci. U.S.A., 82: 86678671 (1985).

8) Pohlman, T. H., Stanness, K. A., Beatty, P. G., Ochs, H. D. and Harlen, J. M.: An endothelial cell surface factor(s) induced in vitro by lipopolysaccharide, interleukin 1 , and tumor necrosis factor increases neutrophil adherence (in part) by a CDw18-dependent mechanism. J. Immunol., 136: 4548-4553 (1986).

9) Schleimer, R. P. and Rutledge, B. K.: Cultured human vascular endothelial cells acquire adhesiveness for neutrophils after stimulation with interleukin 1, endotoxin, and tumor-promoting phorbol diesters. J. Immunol., 136: 649-654 (1986).

10) Dunn, C. J. and Fleming, W. E.: The role of interleukin-1 in the inflammatory response with particular reference to endothelial cell-leukocyte adhesion. In: Kluger, M. J., Oppenheim, J. J., Powanda, M. C., The Physiologic, Metabolic and Immunologic Actions of Interleukin-1. Alan R. Liss, New York, 1985, pp. 45-54.

11) Cavender, D. E., Haskard, D. O., Joseph, B. and Ziff, M.: Interleukin 1 increases the binding of human $\mathrm{B}$ and $\mathrm{T}$ lymphocytes to endothelial cell monolayers. J. Immunol., 136: 203-207 (1986).

12) Haskard, D., Cavender, D., Beatty, P., Springer, T. and Ziff, M.: T lymphocyte adhesion to endothelial cells: Mechanisms demonstrated by antiLFA-1 monoclonal antibodies. J. Immunol., 137: 2901-2906 (1986).

13) Pober, J. S., Bevilacqua, M. P., Mendrick, D. L., Lapierre, L. A., Fiers, W. and Gimbrone, M. A., Jr.: Two distinct monokines, interleukin 1 and tumor necrosis factor, each independently induce biosynthesis and transient expression of the same antigen on the surface of cultured human vascular endothelial cells. J. Immunol., 136: 1680-1687 (1986).

14) Pober, J. S., Gimbrone, M. A., Jr., Lapierre, L. A., Mendrick, D. L., Fiers, W., Rothlein, R. and Springer, T. A.: Overlapping patterns of activation of human endothelial cells by interleukin-1, tumor necrosis factor and immune interferon. J. Immunol., 137: 1893-1896 (1986).

15) Pober, J. S., Collins, T., Gimbrone, M. A., Jr., 
Cotran, R. S., Gitlin, J., Fiers, W., Clayberger, C., Krensky, A., Burakoff, S. J. and Reiss, C. S.: Lymphocytes recognize human vascular endothelial and dermal fibroblast Ia antigens induced by recombinant immune interferon. Nature, 305: 726729 (1983).

16) Cotran, R. S., Gimbrone, M. A., Jr., Bevilacqua, M. P., Mendrick, D. and Pober, J. S.: Induction and detection of a human endothelial activation antigen in vivo. J. Exp. Med., 164 (2): 661-666 (1986).

17) Wheeler, M. R., Bevilacqua, M. P., Luscinskas, F. W., Brock, A. F. and Gimbrone, M. A., Jr.:
Interleukin-1 treated endothelial cells produce an inhibitor of leukocyte-endothelial adhesion. Fed. Proc., 45 (3): 450 (1986).

18) Wheeler, M. E., Luscinskas, F. W., Bevilacqua, M. P. and Gimbrone, M. A., Jr.: Characterization of an endothelial-derived inhibitor of leukocyte adhesion. Fed. Proc., 46 (3): 758 (1987).

19) Wheeler, M. E., Luscinskas, F. W., Bevilacqua, M. P. and Gimbrone, M. A., Jr.: Cultured human endothelial cells stimulated with cytokines or endotoxin produce an inhibitor of leukocyte adhesion. (Submitted for publication) 1987.

\section{Summary}

Studies with cultured human vascular endothelial cells have led to the identification of two endothelial-dependent mechanisms relevant to leukocyte adhesion: the inducible cell surface expression of "endothelial-leukocyte adhesion molecules" (e.g., ELAM-1) and the secretion of a soluble leukocyte adhesion inhibitor (LAI). The interplay of these two mechanisms may contribute to the local regulation of leukocyte-vessel wall interactions in various pathophysiologic settings in vivo. Further understanding of these and related endothelial-dependent mechanisms may provide new insights into the pathogenesis of inflammatory vascular disease processes and potential therapeutic interventions.

\section{Acknowledgments}

The research work summarized above was conducted in collaboration with Drs. Ramzi Cotran, William Luscinskas, Donna Mendrick and Jordan Pober, and was supported by grants from the National Institutes of Health. The technical assistance of A. Brock, K. Case, V. Davis, G. Majeau and D. Smith is gratefully acknowledged. 